\title{
Combinação Convexa de Filtros Adaptativos Para Estimação de Componentes Harmônicos em Sistemas Elétricos de Potência *
}

\author{
Raimundo Nonato Mendes Martins* \\ Francisco das Chagas de Souza* \\ * Laboratório de Sistemas Adaptativos e Processamento de Sinais, \\ Universidade Federal do Maranhão, MA, (e-mail: \\ raimundo_martins@yahoo.com.br; francisco.souza@ufma.br).
}

\begin{abstract}
In this work, we propose a new approach for estimation of harmonic components in power systems based in a convex combination of proportional normalized least-meansquare (PNLMS)-type adaptive filters. In the proposed convex combination, two PNLMStype algorithms are used considering different learning rates and proportionality parameters. Simulation results show that the proposed approach achieves faster convergence, outperforming proportionate adaptive filters used in an individual manner.

Resumo: Neste trabalho, propõe-se uma nova abordagem para estimação de componentes harmônicos em sistemas elétricos de potência baseada em uma combinação convexa de filtros adaptativos do tipo LMS normalizados proporcionais (PNLMS - proportionate normalized leastmean-square). Na combinação convexa proposta, são usados dois algoritmos do tipo PNLMS considerando diferentes taxas de aprendizagem e parâmetros de proporcionalidade. Resultados de simulações mostram que a abordagem proposta alcança convergência mais rápida, superando os filtros adaptativos proporcionais usados individualmente.
\end{abstract}

Keywords: Convex combination; harmonic estimation; adaptive filters; IPNLMS algorithm; electrical power systems.

Palavras-chave: Combinação convexa; estimação de harmônicos; filtros adaptativos; algoritmo IPNLMS; sistemas elétricos de potência.

\section{INTRODUÇÃO}

Os sistemas elétricos de potência têm sido cada vez mais afetados pela presença de componentes harmônicos e interharmônicos. De fato, com o uso crescente de aparelhos eletrônicos, que são cargas não lineares, a presença de frenquências harmônicas nos sistemas elétricos de potência tem aumentado; como consequência, os custos de tais sistemas têm crescido. Essas frequências causam muitos efeitos negativos nos equipamentos conectados ao sistema, como superaquecimento do motor, falhas nos capacitores, mau funcionamento dos relés e erros nos dispositivos de medição (de Souza et al., 2016). Com o objetivo de evitar tais efeitos, os relés de proteção monitoram constantemente parâmetros dos sinais de tensão e corrente, incluindo a frequência, a qual é uma das principais grandezas a ser observada, visto que esta sofrerá alterações incontestáveis durante uma situação faltosa ou indesejada (Barbosa et al., 2008).

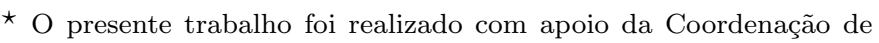
Aperfeiçoamento de Pessoal de Nível Superior - Brasil (CAPES) - Código de Financiamento 001, da Fundação de Amparo à Pesquisa e ao Desenvolvimento Científico e Tecnológico do Maranhão (FAPEMA) e do Conselho Nacional de Desenvolvimento Científico e Tecnológico (CNPq).
}

Para que se tenha planejamento, operação, manutenção e controle dos sistemas elétricos de potência, são necessárias medições precisas e eficientes de frquências harmônicas e interharmônicas. Neste contexto, o rastreamento rápido também é crítico para aplicações como relés de proteção de sub ou sobrefrequência, que exigem estimativas precisas em um período de alguns ciclos ou até mesmo uma fração de um ciclo (Kusljevic, 2008).

Inúmeros métodos de estimação foram propostos para estimar frequências hormônicas no sistema de potência. O método de estimação convencional é baseado na Transformada Rápida de Fourier (FFT) (Sahoo and Subudhi, 2015), transformada discreta de Fourier (DFT), Prony, Método do tipo Newton, Phase-Locked Loop (PLL), redes neurais e filtragem adaptativa, entre outros (Abdollahi et al., 2013).

O espectro dos sinais presentes em sistemas elétricos de potência possui alto grau de esparsidade, pois o mesmo é formado por uma frequência fundamental, a qual detém a maior parte da energia, frequências harmônicas, que são múltiplas da fundamental e interharmônicas. Somente as frequências múltiplas ímpares são consideradas; isto se dá pelo fato de que os sinais oriundos das unidades geradoras são senoidais (Abdollahi et al., 2013). Neste contexto, con- 
siderando que o espectro do sistema elétrico de potência é esparso, filtros adaptativos do tipo LMS normalizado proporcional, como o PNLMS (Proportional Normalized LMS) e IPNLMS (Improved PNLMS) (Benesty and Gay, 2002) podem ser usados para a estimação de frequências harmônicas.

Neste trabalho, propõe-se uma nova abordagem para estimação de frequências harmônicas em sistemas elétricos de potência através de uma combinação convexa de filtros adapativos do tipo LMS normalizado proporcional. Os filtros adaptativos são sintonizados com taxas de aprendizagem e parâmetros de porporcionalidade de modo que um tenha ótimo desempenho no estágio transitório e o outro tenha melhor desempenho no estágio estacionário, fazendo com que este tipo de sintonia seja satisfatória para a combinação convexa. O desempenho da combinação convexa é comparado com o desempenho dos filtros individuais. Este trabalho está organizado da seguinte forma: $\mathrm{Na}$ seção 2 é apresentada a formulação do problema. É apresentado o algoritmo adaptativo IPNLMS, bem como uma métrica para avaliação do grau de esparsidade da resposta ao impulso na seção 3 . A combinação convexa de filtros adaptativos é apresentada na seção 4. As simulações computacionais são apresentadas na seção 5 . Na seção 6 apresentam-se as conclusões.

\section{FORMULAÇÃO DO PROBLEMA}

O sinal de um sistema elétrico de potência pode ser representado, de forma geral, por

$$
y(t)=\sum_{i=1}^{N} A_{i} \operatorname{sen}\left(\omega t+\phi_{i}\right)+v(t)
$$

onde $A_{i}$ e $\phi_{i}$ são a amplitude e fase, respectivamente, dos harmônicos, $N$ representa o total de componentes harmônicos presentes no sinal e $\omega$ é a frequência angular fundamental, $v(t)$ representa um ruído branco gaussiano e $t$, o tempo. Nota-se de (1) que os harmônicos presentes no sinal representam os elementos da série de Fourier desse mesmo sinal.

A forma discreta do sinal representado em (1) é dada por

$$
y(n)=\sum_{i=1}^{N} A_{i} \operatorname{sen}\left(\phi_{\mathrm{i}}\right) \cos \left(\frac{2 i \pi n}{N_{s}}\right)+v(n) .
$$

Em (2), $N_{s}$ é a taxa de amostragem dada por

$$
N_{s}=\frac{f_{s}}{f_{0}}
$$

onde $f_{s}$ e $f_{0}$ são a frequência de amostragem e a frequência fundamental do sinal, respectivamente.

Para a estimação dos harmônicos presentes no sinal dado por (1), pode-se usar um filtro adaptativo, cujo esquema é ilustrado na Figura 1, onde $n$ é o número de iterações, $\mathbf{x}(n)$ é o vetor de entrada, $\mathbf{w}(n)$ é o vetor de pesos do filtro adaptativo, $\hat{y}(n)$ é a saída do filtro, $y(n)$ é o sinal que se deseja estimar e $v(n)$ é um ruído de medição. O sinal de erro é calculado como (Diniz, 2008)

$$
e(n)=y(n)+v(n)-\hat{y}(n)
$$

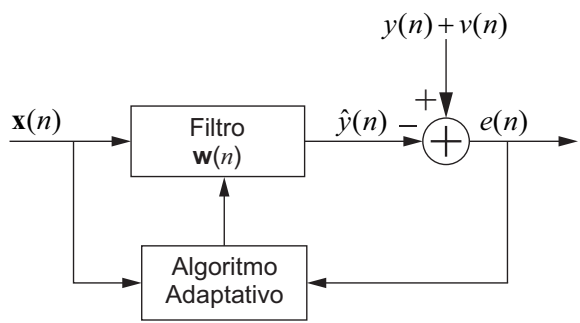

Figura 1. Esquema de filtragem adaptativa.

A Figura 2 mostra um diagrama de filtragem adaptativa aplicada ao problema de estimação de harmônicos em sistemas elétricos de potência, cujo vetor de entrada do filtro adaptativo é representado por

$$
\begin{aligned}
\mathbf{x}(n)= & {\left[\operatorname{sen} \frac{2 \pi n}{N_{s}}, \cos \frac{2 \pi n}{N_{s}}, \cdots,\right.} \\
& \left.\operatorname{sen} \frac{2 \pi N n}{N_{s}}, \cos \frac{2 \pi N n}{N_{s}}\right]^{T},
\end{aligned}
$$

onde o sobrescrito $T$ denota transposta de um vetor ou matriz

Caso um decaimento DC dado por (Sahoo and Subudhi, 2015)

$$
A_{d c} e^{-\beta n T_{s}} \simeq A_{d c}\left(1-\beta n T_{s}\right)
$$

com $\beta$ sendo o fator de decaimento e $T_{s}=2 \pi / \omega N_{s}$, o período de amostragem, seja considerado em (1), então o vetor de entrada pode ser representado por

$$
\begin{aligned}
\mathbf{x}(n)= & {\left[\operatorname{sen} \frac{2 \pi n}{N_{s}}, \cos \frac{2 \pi n}{N_{s}}, \cdots,\right.} \\
& \left.\operatorname{sen} \frac{2 \pi N n}{N_{s}}, \cos \frac{2 \pi N n}{N_{s}}, 1,-n T_{s}\right]^{T}
\end{aligned}
$$

e o vetor de pesos estimados por

$$
\begin{aligned}
\mathbf{w}(n)= & {\left[A_{1} \cos \phi_{1}, A_{1} \operatorname{sen} \phi_{1}, \cdots, A_{N} \cos \phi_{N},\right.} \\
& \left.A_{N} \operatorname{sen} \phi_{N}, A_{d c}, A_{d c} \beta\right]^{T}
\end{aligned}
$$

Ao final da convergência do algoritmo, o vetor de pesos estimados resulta nos coeficientes da série de Fourier e consequentemente as amplitudes e fases estimadas podem ser obtidas a partir, respectivamente, de

$$
A_{i}=\sqrt{\mathrm{w}^{2}(2 i)+\mathrm{w}^{2}(2 i-1)}
$$

e

$$
\phi_{i}=\arctan \left[\frac{\mathrm{w}(2 i)}{\mathrm{w}(2 i-1)}\right]
$$

para $i=1,2, \ldots, N$.

\section{ALGORITMO ADAPTATIVO IPNLMS E GRAU DE ESPARSIDADE}

Nesta seção, aborda-se o algoritmo adaptativo IPNLMS (Improved Proportional Normalized LMS). Além disso, apresenta-se uma métrica para avaliar o grau de esparsidade do espectro estimado pelo filtro adaptativo.

\subsection{Algoritmo Adaptativo PNLMS}

Algoritmos adaptativos do LMS normalizado proporcional (PNLMS) foram desenvolvidos com o objetivo de melhorar o desempenho dos filtros adaptativos para identificação e controle de plantas esparsas. 


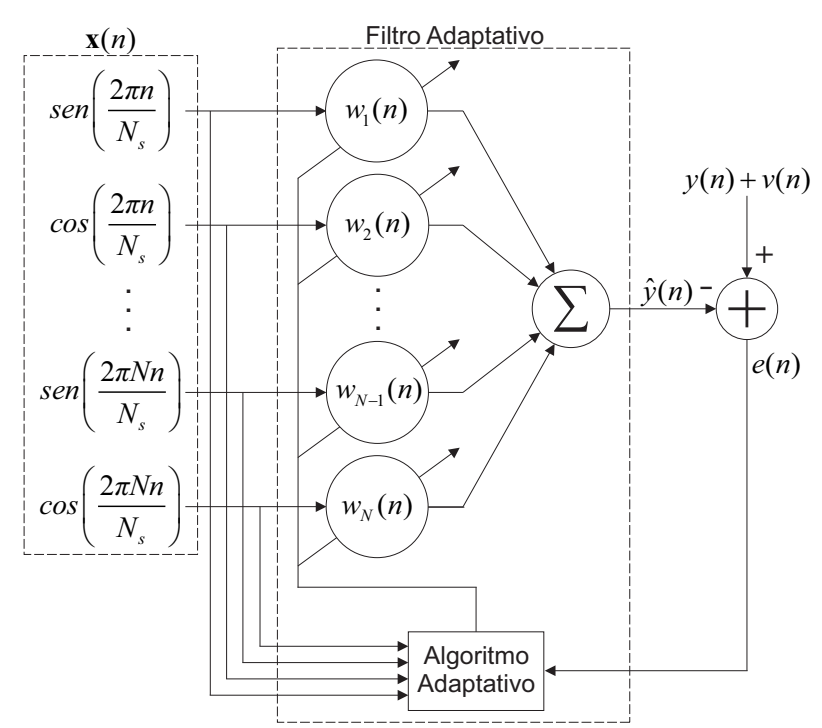

Figura 2. Filtro adaptativo para estimação de harmônicos.

A regra de adaptação dos pesos dos algoritmos normalizados proporcionais é dada pelas expressões (Duttweiler, 2000)

$$
\begin{gathered}
\mathbf{w}(n+1)=\mathbf{w}(n)+\frac{\mu \mathbf{G}(n) e(n) \mathbf{x}(n)}{\mathbf{x}^{T}(n) \mathbf{G}(n) \mathbf{x}(n)+\varepsilon} \\
\mathbf{G}(n)=\operatorname{diag}\left[g_{1}(n) g_{1}(n) \cdots g_{N}(n)\right], \\
g_{i}(n)=\frac{\phi_{i}(n)}{\sum_{j=1}^{N} \phi_{j}(n)}, \\
\phi_{i}(n)=\max \left[f(n),\left|\mathrm{w}_{i}(n)\right|\right]
\end{gathered}
$$

e

$$
f(n)=\rho \max \left[\delta,\|\mathbf{w}(n)\|_{\infty}\right]
$$

onde $e(n)=y(n)-\hat{y}(n)$ é o erro a priori do PNLMS, $y(n)=\mathbf{x}^{T}(n) \mathbf{w}(n)$ é a saída e $\mathbf{x}(n)$ e $\mathbf{w}(n)$ são o vetores de entrada e coeficientes do filtro, respectivamente. A constante $\varepsilon>0$ é um fator de regularização para evitar divisão por 0 e a taxa de aprendizagem, $\mu$, deve ser selecionada no intervalo $0<\mu<2$ para garantir a estabilidade .

\subsection{Algoritmo Adaptativo IPNLMS}

O algoritmo IPNLMS pode ser derivado a partir do PNLMS padrão (de Souza, 2012). Para isso substitui-se $\|\mathbf{w}(n)\|_{\infty}$ por $\|\mathbf{w}(n)\|_{1}$ em (15), que é a norma-1 do vetor de pesos do filtro e adota-se $\rho=1 / N$. Logo, obtém-se um novo fator de ativação dado por

$$
f(n)=\left\{\begin{array}{cc}
\frac{1}{N} \max \left[\delta,\|\mathbf{w}(n)\|_{1}\right], & n=0 \\
\frac{\|\mathbf{w}(n)\|_{1}}{N}, & n \geq 1 .
\end{array} .\right.
$$

Desta forma, para $n \geq 1$, a função de proporcionalidade dada por (14) pode ser reescrita da forma

$$
\phi_{i}(n)=\left[\frac{\|\mathbf{w}(n)\|_{1}}{N},\left|\mathrm{w}_{i}(n)\right|\right] .
$$

Agora, para a função de proporcionalidade dada por (17), uma média ponderada é usada no lugar do operador $\max [\cdot]$. Logo, a nova função de proporcionalidade é (Benesty and Gay, 2002)

$$
\phi_{i}(n)=(1-\alpha) \frac{\|\mathbf{w}(n)\|_{1}}{N}+(1+\alpha)\left|\mathrm{w}_{i}(n)\right|,
$$

onde $-1 \leq \alpha<1$ é um fator de ponderação. De (18) notase que a primeira parcela do lado direito é comum a todos os pesos do filtro, enquanto que a segunda é proporcional à magnitude do $i$-ésimo peso.

Considerando (18), desenvolve-se o denominador de (13), entao fica

$$
\begin{aligned}
\sum_{i=1}^{N} \phi_{i}(n) & =\sum_{i=1}^{N}\left[(1-\alpha) \frac{\|\mathbf{w}(n)\|_{1}}{N}+(1+\alpha)\left|\mathrm{w}_{i}(n)\right|\right] \\
& =\left[\frac{1-\alpha}{N} \sum_{i=1}^{N}\|\mathbf{w}(n)\|_{1}\right] \\
& +\left[(1+\alpha) \sum_{i=1}^{N}\left|\mathrm{w}_{i}(n)\right|\right] \\
& =\frac{1-\alpha}{N} N\|\mathbf{w}(n)\|_{1}+(1+\alpha)\|\mathbf{w}(n)\|_{1} \\
& =(1-\alpha)\|\mathbf{w}(n)\|_{1}+(1+\alpha)\|\mathbf{w}(n)\|_{1} \\
& =2\|\mathbf{w}(n)\|_{1}
\end{aligned}
$$

Agora, substituindo (18) e (19) em (13), obtém-se o ganho individual do algoritmo IPNLMS dado por

$$
g_{i}(n)=(1-\alpha) \frac{1}{2 N}+(1+\alpha) \frac{\left|\mathrm{w}_{i}(n)\right|}{2\|\mathbf{w}(n)\|_{1}+\varsigma},
$$

onde $\varsigma>0$ é um parâmetro de regularização usado para evitar divisão por zero. O fator $\alpha$ é denominado parâmetro de proporcionalidade do algoritmo IPNLMS.

\subsection{Grau de Esparsidade}

Um vetor ou uma matriz são esparsos, se grande parte de seus elementos são nulos. Em outras palavras, a esparsidade é uma característica inerente a vetores e matrizes (Branco, 2016). Seja o vetor

$$
\mathbf{p}=\left[p_{1}, p_{2}, p_{3}, \cdots, p_{N}\right]^{T}
$$

definido em um dado subespaço de $\mathbb{R}^{N}$. O vetor $\mathbf{p}$ terá espasidade elevada se a grande maioria de seus elementos, $p_{i}$, com $i=1,2, \cdots, N$, é nula.

O grau de esparsidade de uma planta é calculado de acordo com (Huang et al., 2006)

$$
S(\mathbf{p})=\frac{N}{N-\sqrt{N}}\left(1-\frac{\|\mathbf{p}\|_{1}}{\sqrt{N}\|\mathbf{p}\|_{2}}\right),
$$

onde $\|\mathbf{p}\|_{1}$ e $\|\mathbf{p}\|_{2}$ sao a norma-1 e a norma-2, respectivamente, do vetor de coeficientes da planta p. Quanto mais próximo $S(\mathbf{p})$ estiver de 1 , mais esparsa a planta é considerada, no outro sentido, quando mais próximo de 0 , menos esparsa a planta é considerada, isto é, o grau de esparsidade é restrito ao intervalo $0 \leq S(\mathbf{p}) \leq 1$. A variável $N$ indica o número de coeficientes de $\mathbf{p}$.

\section{COMBINAÇÃO CONVEXA DE FILTROS ADAPTATIVOS}

A combinação de filtros adaptativos explora o princípio de "dividir e conquistar". Tal princípio tem sido explorado 
com sucesso pela comunidade de aprendizado de máquina (Arenas-Garcia et al., 2016).

O esquema de combinação mais simples é feito com apenas dois filtros adaptativos como mostrado na Figura 3, na qual tem-se o sinal a ser estimado dado por

$$
y(n)=\mathbf{x}^{T}(n) \mathbf{w}_{o}(n)+v(n),
$$

onde $\mathbf{x}(n), \mathbf{w}_{o}(n)$ e $v(n)$ são o vetor de entrada definido em (5), o vetor de pesos ótimos e um ruído de medição, respectivamente.

A saída da combinação é dada por

$$
\hat{y}(n)=\lambda(n) \hat{y}_{1}(n)+[1-\lambda(n)] \hat{y}_{2}(n),
$$

onde $\hat{y}_{i}(n)=\mathbf{x}^{T}(n) \mathbf{w}_{i}(n)$, com $i=1,2$, são as saídas dos dois filtros adaptativos caracterizados por seus vetores de pesos $\mathbf{w}_{i}(n)$, e $\lambda(n)$ é o parâmetro de mistura. Similarmente, o vetor de peso estimado, o erro e o erro a priori do esquema de combinação são dados por

$$
\begin{gathered}
\mathbf{w}(n)=\lambda(n) \mathbf{w}_{1}(n)+[1-\lambda(n)] \mathbf{w}_{2}(n) \\
e(n)=\lambda(n) e_{1}(n)+[1-\lambda(n)] e_{2}(n) \\
e_{a}(n)=\lambda(n) e_{a, 1}(n)+[1-\lambda(n)] e_{a, 2}(n),
\end{gathered}
$$

onde $e_{i}(n)=y(n)-\hat{y}_{i}(n)$, com $i=1,2$. O Parâmetro de mistura, $\lambda(n)$ é restrito ao intervalo $[0,1]$ para combinações convexas, enquanto que para combinações afins pode ter qualquer valor real (Arenas-Garcia et al., 2016).

Cada filtro componente é adaptado usando suas próprias regras e erros, enquanto que o parâmetro $\lambda(n)$ é escolhido para minimizar o erro quadrático geral da combinação.

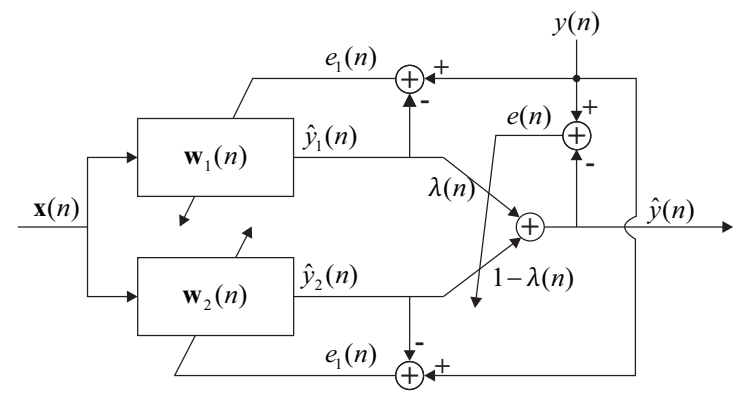

Figura 3. Combinação convexa de dois filtros adaptativos.

Recorre-se a uma função de ativação para manter o parâmetro de mistura dentro do intervalo de interesse. Usa-se uma função sigmoidal com adaptação de um parâmetro auxiliar $a(n)$ dado por

$$
\lambda(n)=\operatorname{sgm}[a(n)]=\frac{1}{1+e^{-a(n)}} .
$$

Para adaptação do parâmetro de mistura, $\lambda(n)$, usa-se o método do gradiente estocástico para minimizar o erro $a$ priori total da combinação, $e(n)$. Logo,

$$
\begin{gathered}
e^{2}(n)=[y(n)-\hat{y}(n)]^{2} \\
a(n+1)=a(n)-\frac{\mu_{a}}{2} e(n) \frac{\partial e^{2}(n)}{\partial a(n)} \\
a(n+1)=a(n)+\mu_{a} e(n)\left[\hat{y}_{1}(n)\right. \\
\left.-\hat{y}_{2}(n)\right] \lambda(n)[1-\lambda(n)] .
\end{gathered}
$$

Uma outra forma de adaptar o parâmetro auxiliar é recorrer a uma estimação da potência de $\left[\hat{y}_{1}(n)-\hat{y}_{2}(n)\right]$ dada por

$$
p(n+1)=v p(n)+(1-v)\left[\hat{y}_{1}(n)-\hat{y}_{2}(n)\right]^{2},
$$

onde $v$ funciona como um fator de esquecimento que pode ser facilmente ajustado, por exemplo, $v=0,9$ (AzpicuetaRuiz et al., 2008).

Logo a adaptação do parâmetro auxiliar fica

$$
\begin{aligned}
a(n+1) & =a(n)+\frac{\mu_{a}}{p(n)} e(n)\left[\hat{y}_{1}(n)\right. \\
& \left.-\hat{y}_{2}(n)\right] \lambda(n)[1-\lambda(n)] .
\end{aligned}
$$

\section{SIMULAÇÕES}

Para avaliar o desempenho da combinação convexa proposta, são realizadas simulações de Monte Carlo (média de 100 realizações) aplicadas na estimação de harmônicos em sistemas elétricos de potência, considerando-se dois cenários (um com dois harmônicos ímpares e outro com sete harmônicos ímpares). Nas simulações são combinados dois algoritmos adaptativos IPNLMS.

A figura de mérito considerada para avaliar a velocidade de convergência dos algoritmos e da combinação convexa é o desalinhamento normalizado em $\mathrm{dB}$ dado por

$$
\Psi(n)=10 \log _{10}\left(\frac{\left\|\mathbf{w}_{\mathbf{o p t}}-\mathbf{w}(n)\right\|_{2}^{2}}{\left\|\mathbf{w}_{\mathbf{o p t}}\right\|_{2}^{2}}\right),
$$

onde $\mathbf{w}_{\text {opt }}$ é obtido a partir de (8) e (34) e w $(n)$ é o vetor de pesos estimados ao final da convergência dos algoritmos.

\subsection{Cenario 1}

Neste cenário é considerado um sinal dado por

$$
\begin{gathered}
y(t)=1,2 \operatorname{sen}(\omega t+\pi / 6)+0,8 \operatorname{sen}(3 \omega t+\pi / 3) \\
+0,3 \operatorname{sen}(5 \omega t+\pi / 4)+0,05 e^{-0,2 t} .
\end{gathered}
$$

As frequências fundamental e de amostragem são $60 H z$ e $1500 \mathrm{~Hz}$, respectivamente. É considerado um ruído aditivo branco gaussiano com SNR de $30 \mathrm{~dB}$. A Figura 4 mostra o sinal dado em (34) e o espectro de frequência estimado é mostrado na Figura 5. Nota-se ainda da Figura 5 que o grau de esparsidade é elevado e seu valor calculado por (22) é 0,96561 . Para o filtro adaptativo IPNLMS 1 são definidos $\mu_{1}=0,17$ e $\alpha_{1}=-0,6$ para o fator de passo e parâmetro de proporcionalidade, respectivamente. Para o IPNLMS 2 o fator de passo e o parâmetro de proporcionalidade são $\mu_{2}=0,85$ e $\alpha_{2}=-0,9$, respectivamente. Para a combinação convexa são definidos um fator de esquecimento, $v=0,9$ e fator de passo do parâmetro auxiliar, $\mu_{a}=6$.

A Figura 6 mostra uma comparação de desempenhos dos algoritmos LMS, NLMS e IPNLMS com os seguintes parâmetros: taxa de aprendizagem, $\mu_{1}=0,199$ para $\mathrm{o}$ LMS, taxa de aprendizagem, $\mu_{2}=0,199$ para o NLMS e taxa de aprendizagem, $\mu_{3}=0,19$ e fator de proporcionalidade, $\alpha=0$ para o IPNLMS. A Figura 7 mostra o desalinhamento normalizado em dB dos algoritmos bem como da combinação convexa. Nota-se ainda da Figura 7 que a combinação apresenta desempenho superior em relação aos algoritmos combinados. Pode ser notado que a combinação convexa rastreia o algoritmo mais rapido 
no regime transitório e em seguida rastreia o algoritmo que tem melhor desempenho no regime estacionário. A evolução do parâmetro de mistura pode ser visto na Figura 8. A estimação da amplitude e fase do 3 o harmônico são mostradas na Figura 9(a) e na Figura 9(b), respectivamente. A amplitude e fase do 5o harmônico podem ser vistas, respectivamente, nas Figuras 10(a) e 10(b). Nota-se das Figuras 9 a 10 que as amplitudes e fases são estimadas em dois ciclos da frequência fundamental.

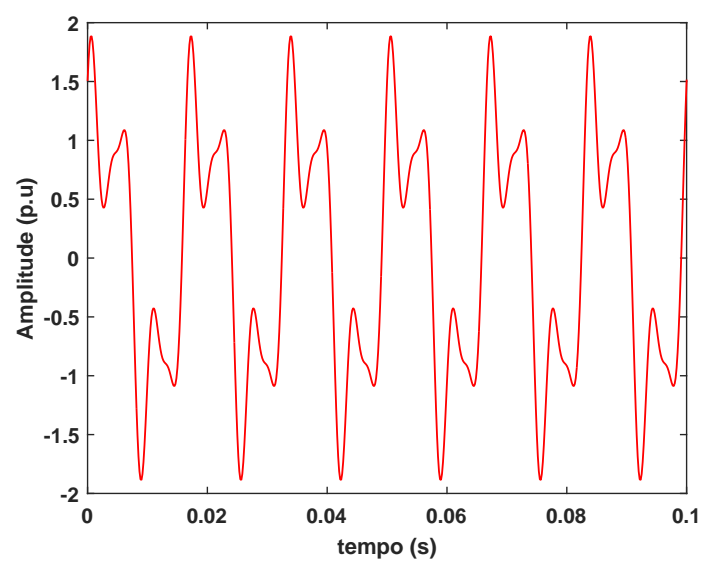

Figura 4. Forma de onda do sinal dado em (34).

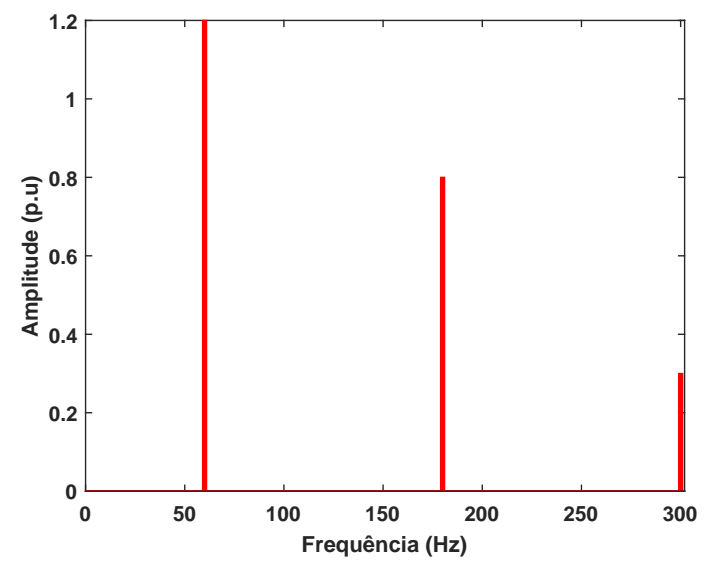

Figura 5. Esparsidade do espectro de frequência estimado, considerando uma resolução de valor $1 \mathrm{~Hz}$. Grau de esparsidade $S(\mathbf{w})=0,96561$.

\subsection{Cenario 2}

Seja um sinal elétrico dado por

$$
\begin{aligned}
y(t) & =\operatorname{sen}(\omega t+0,147)+0,1 \operatorname{sen}(3 \omega t+0,349) \\
& +0,08 \operatorname{sen}(5 \omega t+0,523)+0,08 \operatorname{sen}(9 \omega t+0,693) \\
& +0,06 \operatorname{sen}(11 \omega t+0,872)+0,05 \operatorname{sen}(13 \omega t+0,047) \\
& +0,03 \operatorname{sen}(19 \omega t+0,221+0,01 \operatorname{sen}(25 \omega t+0,396) \\
& +0,05 e^{-0,2 t}
\end{aligned}
$$

Para este sinal são considerados os valores de $60 \mathrm{~Hz}$ e $3.200 \mathrm{~Hz}$ para frequência fundamental e frequência de amostragem, respectivamente. É considerado um ruído aditivo branco gaussiano com SNR de $30 \mathrm{~dB}$. O sinal dado em (35) é mostrado na Figura 11 e o espectro de frequência

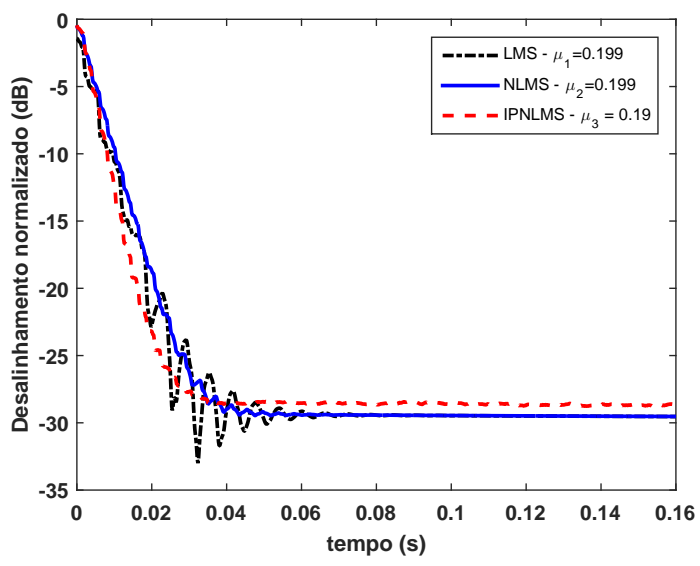

Figura 6. Comparação de desempenhos dos algoritmos LMS $\left(\mu_{1}=0.199\right)$, NLMS $\left(\mu_{2}=0.199\right)$ e IPNLMS $\left(\mu_{3}=0.19\right.$ e $\left.\alpha=0,0\right)$, considerando o cenário 1 .

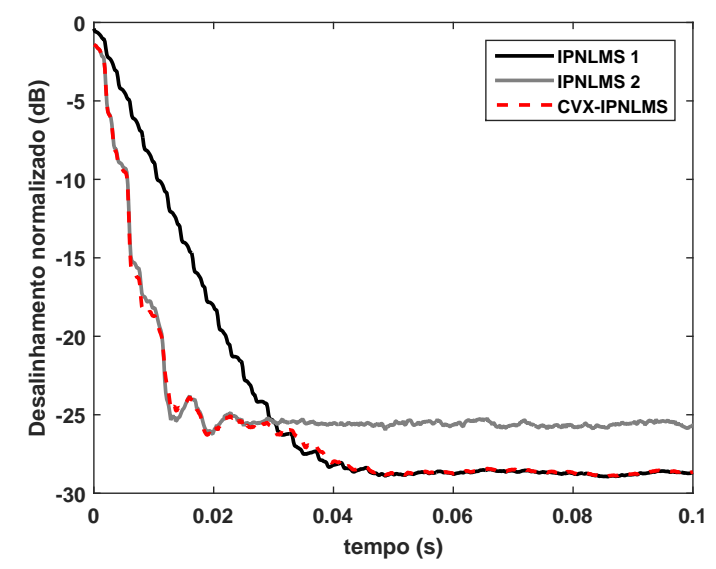

Figura 7. Desempenho dos algoritmos IPNLMS $1\left(\mu_{1}=\right.$ $\left.0,17, \alpha_{1}=-0,6\right)$, IPNLMS $2\left(\mu_{2}=0,85, \alpha_{2}=-0,9\right)$ e CVX-IPNLMS $\left(\mu_{a}=6, v=0,9\right)$.

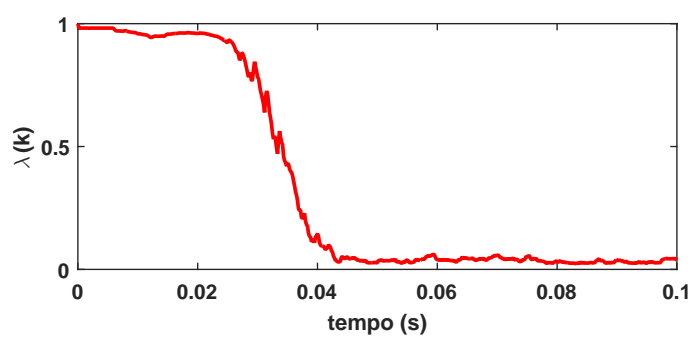

Figura 8. Parâmetro de mistura da combinação convexa para o cenário 1.

estimado pode ser visualizado na Figura 12. O grau de esparsidade para esse espectro é 0,98968. Para o filtro adaptativo IPNLMS 1 tem-se o fator de passo, $\mu_{1}=0,3$, e parâmetro de proporcionalidade $\alpha_{1}=-0,75$, enquanto que para o filtro adaptativo IPNLMS 2 são definidos os parâmetros $\mu_{2}=0,95$ e $\alpha_{2}=-0,78$ para o fator de passso e parâmetro de proporcionalidade, respectivamente. Para a combinação convexa o fator de esquecimento foi definido com valor de $v=0,9$ e passo para o parâmetro auxiliar, $\mu_{a}=6$. 


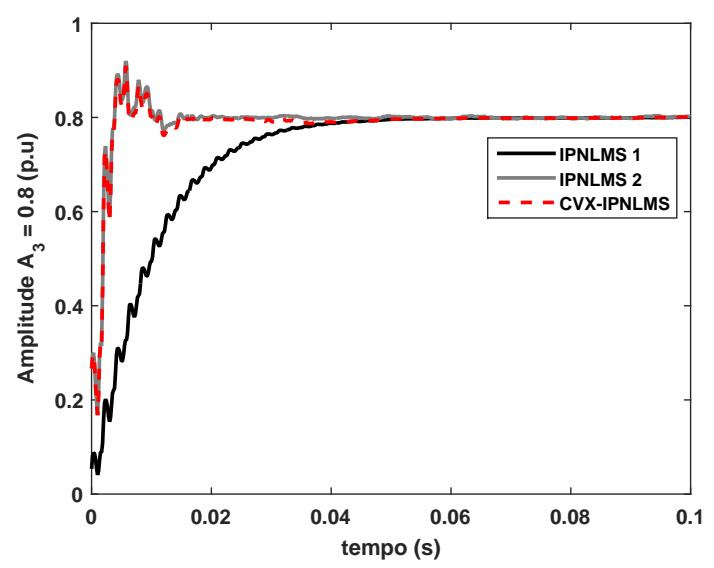

(a)

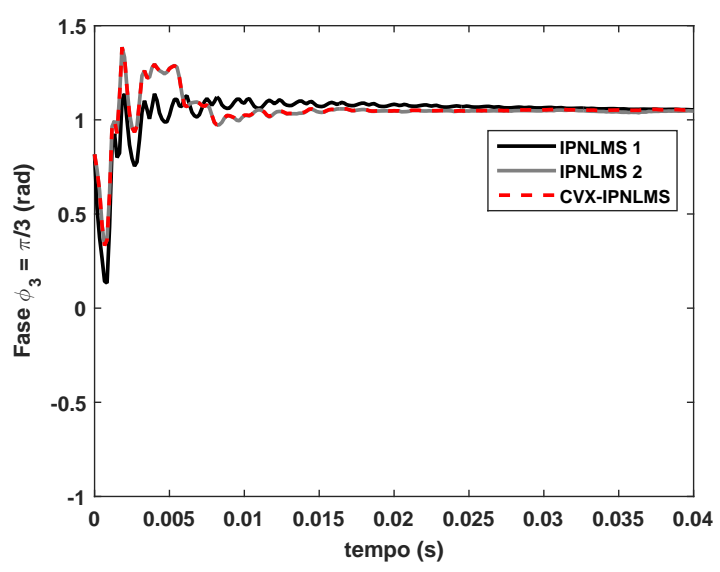

(b)

Figura 9. Estimação do 3 o harmônico do cenário 1. (a) Amplitude em p.u. (b) Fase em radiano.

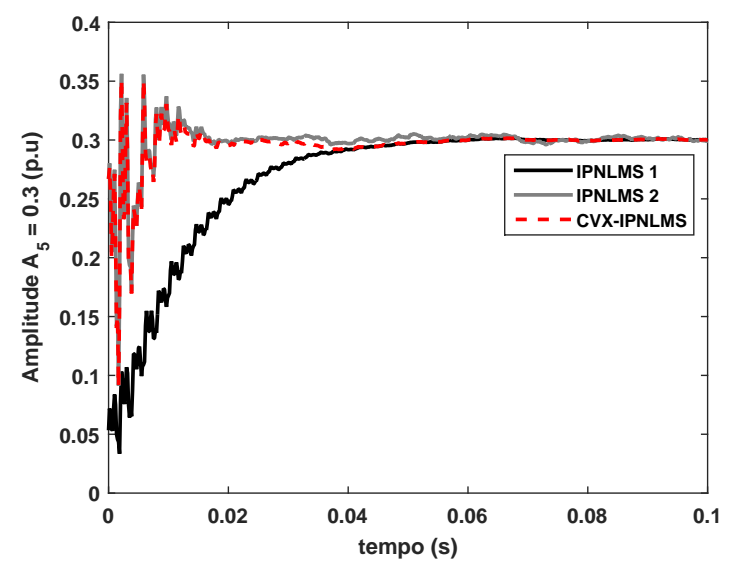

(a)

A Figura 11 mostra a forma de onda do sinal dado em (35), enquanto que o espectro esimado é mostrado na Figura 12. Ainda da Figura 12 pode ser observado que o grau de esparsidade é 0,98968 .

A amplitude estimada bem como a fase do 3o harmônico são mostradas na Figura 15. Na figura 16, podem ser vistas a amplitude e fase estimadas do 5 o harmônico. Observa-se das Figuras 15 a 16 que os harmônicos são estimados em dois ciclos da frequência fundamental.

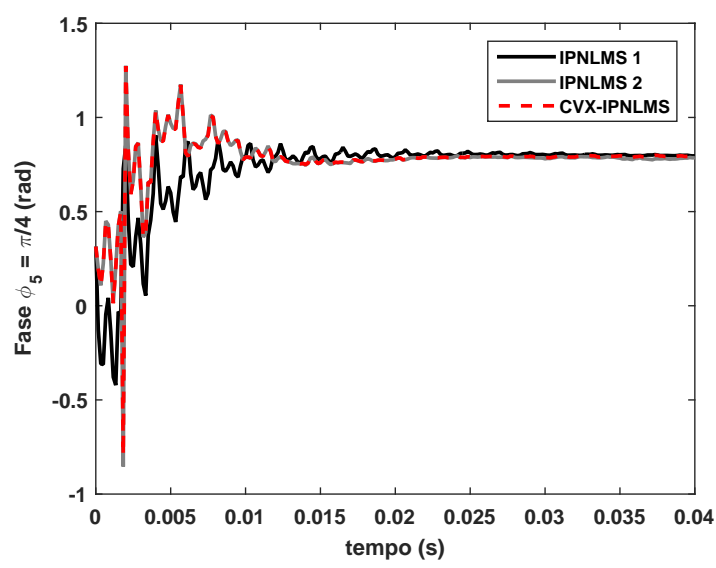

(b)

Figura 10. Estimação do 5o harmônico do cenário 1. (a) Amplitude em p.u. (b) Fase em radiano.

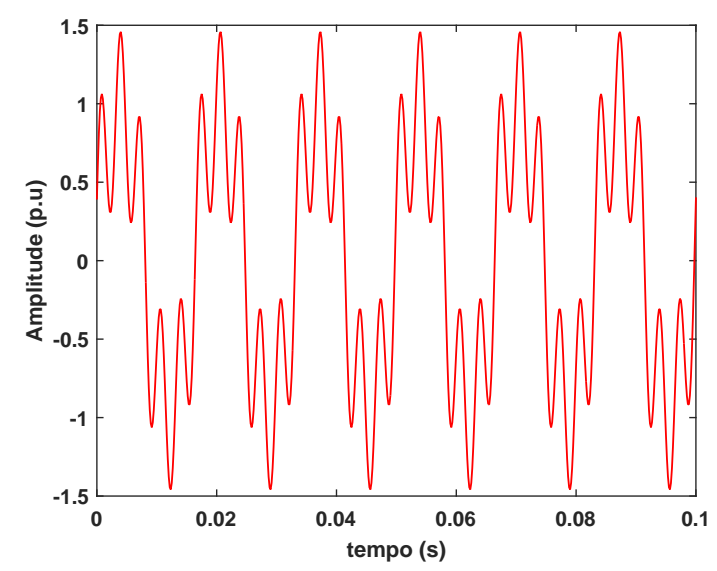

Figura 11. Forma de onda do sinal dado em (35).

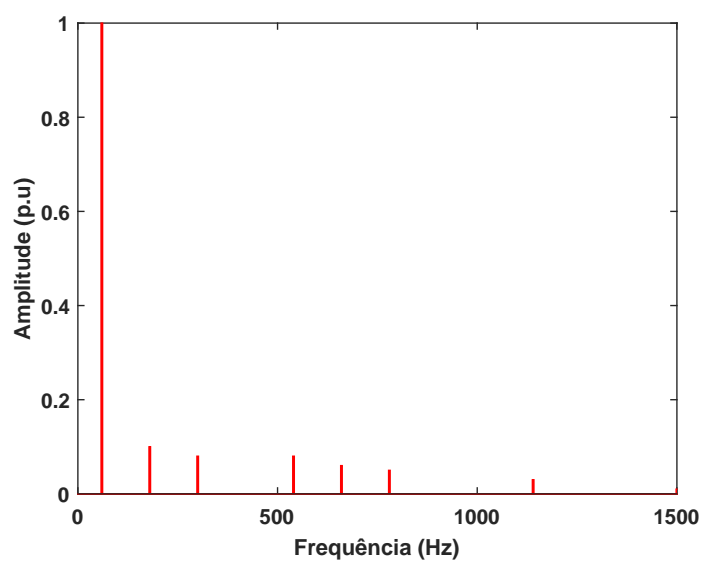

Figura 12. Grau de esparsidade $S(\mathbf{w})=0,98968$ do espectro estimado com resolução $1 \mathrm{~Hz}$.

O desempenho dos algoritmos bem como da combinação convexa são mostrados na Figura 13. Percebe-se da Figura 13 que a combinação apresenta um desempenho superior em relação aos algoritmos adaptativos combinados. A Figura 14 mostra o a evolução do parâmetro de mistura da combinação convexa. 


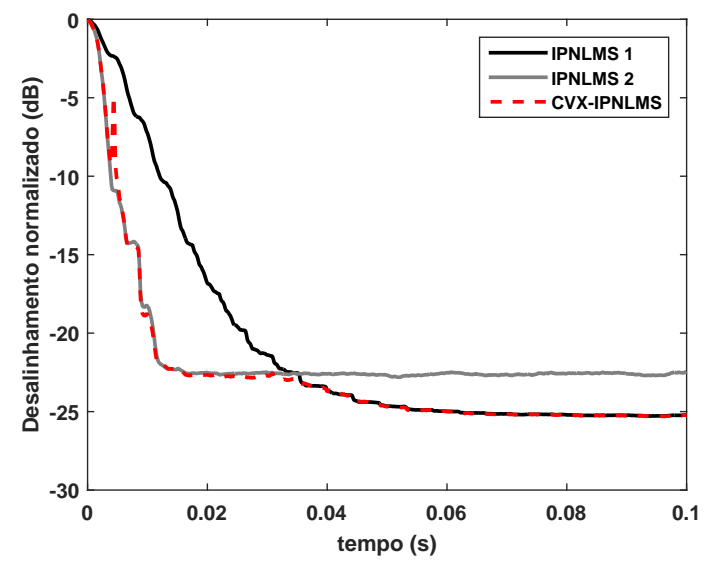

Figura 13. Desempenho dos algoritmos IPNLMS $1\left(\mu_{1}=\right.$ $\left.0,3, \alpha_{1}=-0,75\right)$, IPNLMS $2\left(\mu_{2}=0,95, \alpha_{2}=\right.$ $-0,78)$ e CVX-IPNLMS $\left(\mu_{a}=6, v=0,9\right)$.

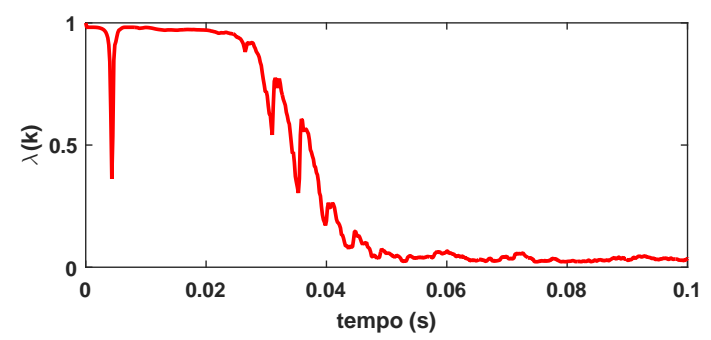

Figura 14. Parâmetro de mistura da combinação convexa para o cenário 2 .

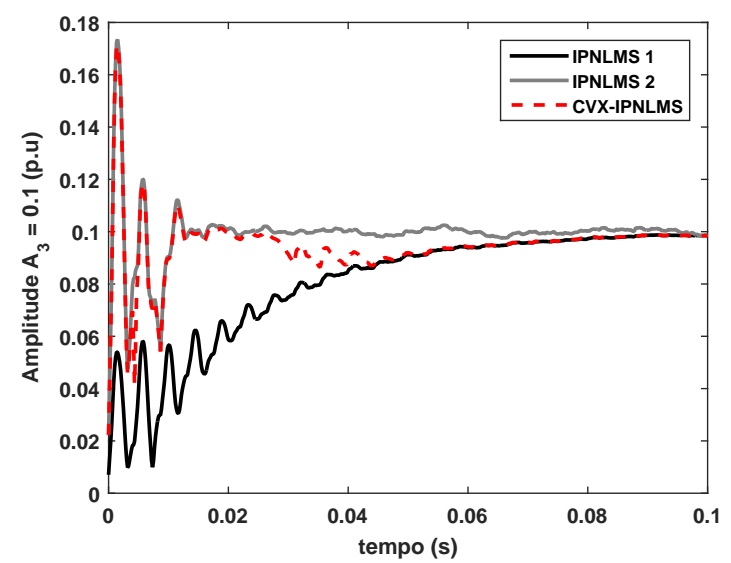

(a)

\section{CONCLUSÃO}

Neste trabalho, propõe-se uma nova abordagem para estimação de harmônicos em sistemas elétricos de potência. Para este fim, utiliza-se uma combinação convexa de filtros adaptativos do tipo LMS normalizado proporcional (PNLMS - proportionate normalized least-mean-square). Para os diferente cenários considerados, os resultados mostram que a abordagem proposta alcança convergência mais rápida, superando os filtros adaptativos usados de forma convencional. Além disso, devido à característica esparsa

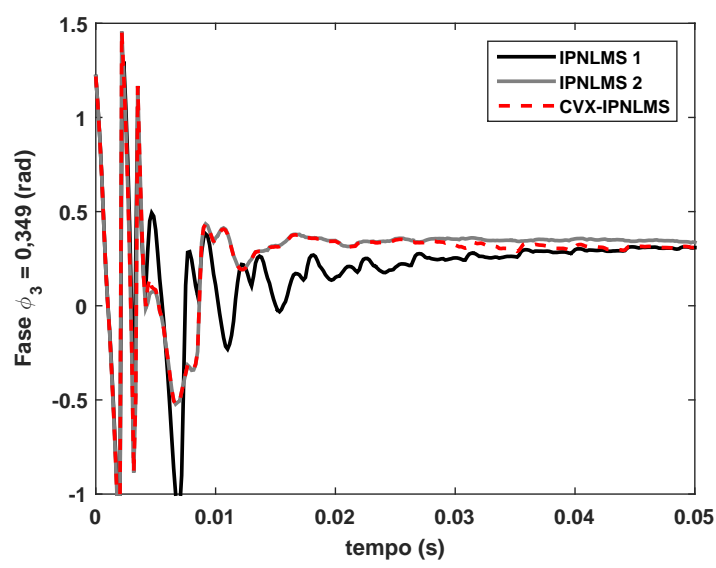

(b)

Figura 15. Estimação do 3o harmônico do cenário 2. (a) Amplitude em p.u. (b) Fase em radiano.

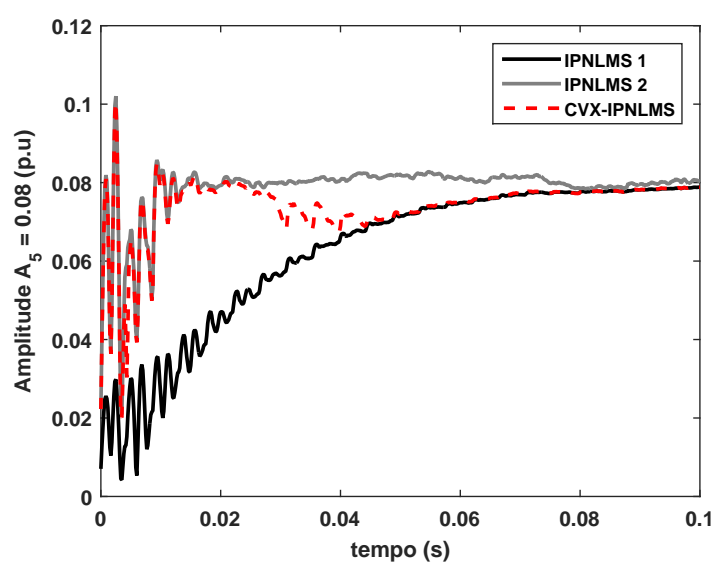

(a)

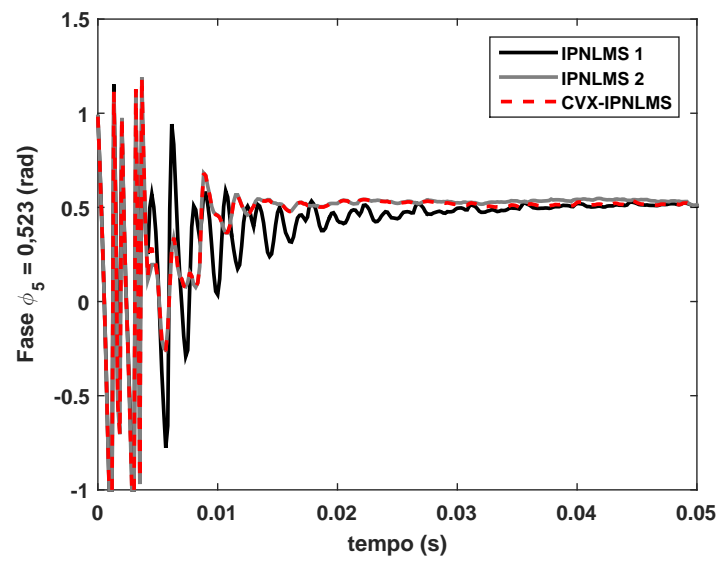

(b)

Figura 16. Estimação do 5o harmônico do cenário 2. (a) Amplitude em p.u. (b) Fase em radiano.

deste tipo de aplicação (estimação de harmônicos), os resultados das simulações mostram que os algoritmos do tipo PNLMS apresentam melhor desempenho em termos de velocidade de convergência quando comparado com os algoritmos LMS e NLMS. 


\section{AGRADECIMENTOS}

Os autores agradecem a Coordenação de Aperfeiçoamento de Pessoal de Nível Superior - Brasil (CAPES) - Código de Financiamento 001, a Fundação de Amparo à Pesquisa e ao Desenvolvimento Científico e Tecnológico do Maranhão (FAPEMA) e ao Conselho Nacional de Desenvolvimento Científico e Tecnológico ( $\mathrm{CNPq})$.

\section{REFERÊNCIAS}

Abdollahi, A., Zhang, P., Xue, H., and Li, S. (2013). Enhanced subspace-least mean square for fast and accurate power system measurement. IEEE Transactions on Power Delivery, 28(1), 383-393.

Arenas-Garcia, J., Azpicueta-Ruiz, L.A., Silva, M.T., Nascimento, V.H., and H, S.A. (2016). Combinations of adaptive filters: performance and convergence properties. IEEE Signal Processing Magazine, 33(1), 120-140.

Azpicueta-Ruiz, L.A., Figueiras-Vidal, A.R., and ArenasGarcia, J. (2008). A normalized adaptation scheme for the convex combination of two adaptive filters. In Acoustics, Speech and Signal Processing, 2008. ICASSP 2008. IEEE International Conference on, 3301-3304. IEEE.

Barbosa, D., Monaro, R.M., Coury, D.V., and Oleskovicz, M. (2008). Filtragem adaptativa para a estimação da freqüência em sistemas elétricos de potência. Sba: Controle $\mathcal{E}$ Automação Sociedade Brasileira de Automatica, 19(2), 226-234.

Benesty, J. and Gay, S.L. (2002). An improved pnlms algorithm. Orlando, FL, USA, 1881-1884.

Branco, C.A.S.C. (2016). Algoritmos adaptativos lms normalizados proporcionais: proposta de novos algoritmos para identificação de plantas esparsas.

de Souza, F.d.C. (2012). Algoritmos adaptativos lms normalizados proporcionais: proposta de um novo algoritmo e sua modelagem estocástica.

de Souza, M.Z., Pereira, M., Silveira, P., and Ribeiro, P. (2016). A matlab tool to study harmonics penetration in grid unbalanced conditions. In Harmonics and Quality of Power (ICHQP), 2016 17th International Conference on, 715-720. IEEE.

Diniz, P.S. (2008). Adaptive filtering: algorithms and practical implementation. The international series in Engineering and Computer Scienc, 23-50.

Duttweiler, D.L. (2000). Proportionate normalized leastmean-squares adaptation in echo cancelers. IEEE Transactions on Speech and Audio Processing, 8(5), 508-518.

Huang, Y., Benesty, J., and Chen, J. (2006). Acoustic MIMO signal processing. Springer Science \& Business Media.

Kusljevic, M.D. (2008). A simple method for design of adaptive filters for sinusoidal signals. IEEE Transactions on Instrumentation and Measurement, 57(10), 2242-2249.

Sahoo, H.K. and Subudhi, U. (2015). Adaptive estimation of power system harmonics and decaying dc using volterra rls. In 2015 IEEE Power, Communication and Information Technology Conference (PCITC), 43-47. IEEE. 